\title{
Prevalence of shoulder pain in the community: the influence of case definition
}

\author{
Daniel P Pope, Peter R Croft, Christina M Pritchard, Alan J Silman
}

Arthritis and

Rheumatism Councils'

Epidemiology

Research Unit,

The University of

Manchester,

Manchester

D P Pope

C M Pritchard

A J Silman

Industrial and Community Health

Research Centre,

School of Postgraduate

Medicine,

University of Keele,

Stoke on Trent

P R Croft

Correspondence to:

Dr D P Pope, Arthritis and

Rheumatism Councils'

Epidemiology Research Unit,

The University of

Manchester, Stopford

Building, Oxford Road,

Manchester M13 9PT.

Accepted for publication

20 February 1997
Abstract

Objective-To compare estimates of the occurrence of shoulder pain according to (a) different approaches to defining 'shoulder' and (b) restricting the definition to only include those with associated disability.

Methods-A postal questionnaire survey was sent to a sample of 500 patients registered with a general practice in south Manchester. After additional mailings to non-responders, 312 questionnaires were returned (66\% adjusted response rate). Four definitions of shoulder pain were used to estimate the occurrence of symptoms derived from answers to the questionnaire. Two were based on questions asking directly about pain in the shoulder and the upper trunk and neck region respectively and two were based on markings on a pain drawing in the shoulder complex and the upper trunk respectively. To determine the occurrence of disabling shoulder pain responders were subsequently approached for interview. Of the responders, 232 (74\%) were successfully interviewed. Those indicating that they were suffering from 'current' shoulder symptoms, pain on the day of interview, were asked to complete a short, 23 item, questionnaire enquiring about disability in daily living associated with such symptoms.

Results-In total $160 \quad(51 \%)$ people reported shoulder pain according to at least one definition. This one month period prevalence ranged from $31 \%$ to $48 \%$ across the four definitions with the lowest estimate being for the question asking directly about shoulder symptoms. In total 84 people ( $27 \%$ of all respondents) answered positively to all four definitions. Only seven people who answered positively when asked directly about shoulder pain did not indicate symptoms on the pain drawing in the shoulder complex. By contrast $65(30 \%)$ of those answering negatively to the direct question about shoulder pain indicated symptoms on the pain drawing in the upper trunk region or answered positively to the direct question about pain in the upper trunk or neck region. However only $19(9 \%)$ of those answering negatively to the direct question indicated symptoms in the shoulder complex on the pain drawing, compared with $38(18 \%)$ indicating symptoms in the upper trunk region and 59
$(27 \%)$ symptoms in the upper trunk and neck region. Limiting the definition to only include current symptoms with some associated disability (at least one item on the disability questionnaire being answered positively) restricted the point prevalence to $20 \%(n=46)$.

Conclusions-Using a pain drawing based definition with case ascertainment restricted to an area in and around the shoulder complex is recommended for surveys assessing the occurrence of shoulder symptoms in the general population. To solve the problem of the poor specificity associated with symptom based definitions it is useful to incorporate an additional classification to restrict the definition to more disabling problems.

(Ann Rheum Dis 1997;56:308-312)

Shoulder pain is recognised as a disabling problem and is a frequent reason for consultation in general practice with approximately $1 \%$ of adults in the UK consulting with a new episode of shoulder pain in the course of one year. ${ }^{1}$ In the community as many as $20 \%$ of the adult population experience shoulder symptoms at any one time, many of whom do not consult their doctor, ${ }^{2}$ and these complaints seem to be increasing in incidence. ${ }^{3}$ It is important to investigate shoulder pain in the community to understand the full impact such complaints have on the general population.

Defining shoulder symptoms for epidemiological studies of occurrence presents a number of difficulties. The complex interrelations between the shoulder and adjacent areas and the frequent occurrence of referred pain make clinical case definition problematic. ${ }^{4}$ Defining shoulder pain in terms of separate anatomical diagnoses requires detailed physical examination, ${ }^{5}$ which may be impractical for large scale epidemiological studies but, more importantly, has questionable repeatability and validity. ${ }^{6}$ In practice, therefore, 'shoulder pain' has been applied as an all encompassing term in studies of its occurrence.

The classification of a case of 'shoulder pain' has been approached in various ways in studies of its occurrence. Many studies have asked directly about the presence of pain in the shoulder. $^{78}$ This relies, however on the respondents' perceptions as to the anatomical origin of their symptoms. Pains arising from structures around the shoulder complex are often felt in a wider area, for example the neck, upper arm or upper trunk, ${ }^{9}$ and thus may be undetected with such a 'self perceived' 
(1) 'During the past month, have you experienced pain in your shoulder(s) lasting more than 24 hours?'

(2) Pain felt in and around the shoulder complex
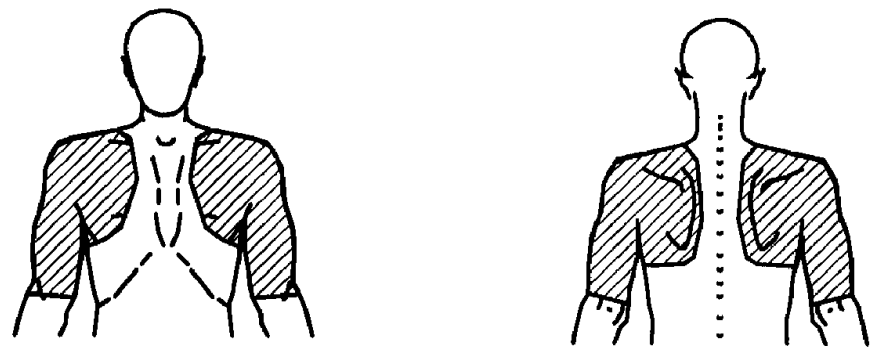

(3) Pain felt in the 'upper trunk' region
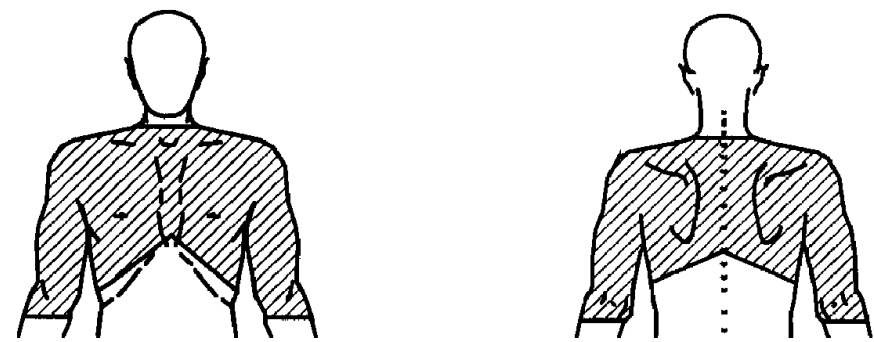

Figure 1 Case definitions of shoulder pain used in the cross sectional survey.

definition. Alternatively pain drawings or manikins have been used. ${ }^{10}$ These require the respondent to indicate the presence or absence of symptoms on a line drawing of the body and have the advantage that they can be independently classified. Referred symptoms originating outside the shoulder complex, however, might artificially inflate prevalence estimates based on such a definition. To date, there has been no research considering how these different approaches affect estimates of the prevalence of shoulder pain.

In addition, crude estimates of shoulder pain occurrence based on self reported pain may give a misleading perspective on the public health burden of shoulder disorders. Thus it could be argued that studies reporting the prevalence estimates of shoulder pain to be one in five of the population ${ }^{11}$ would presumably have included many minor or transient symptoms. Studies of the occurrence of shoul-

(4) Symptoms in a pre-shaded area (upper trunk and chest)
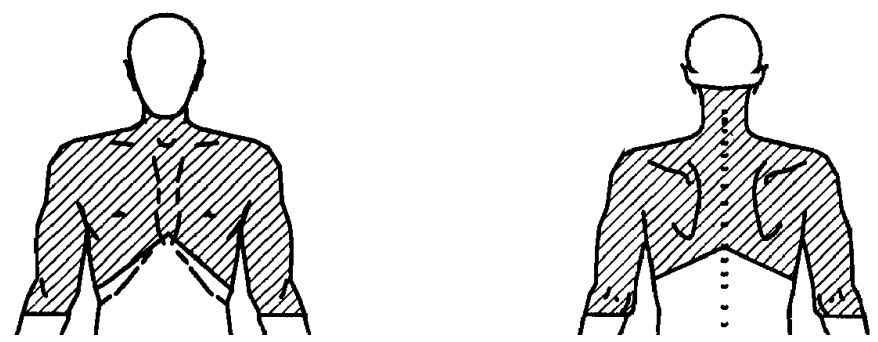

Figure 2 Case definition of shoulder pain used in the cross sectional survey. der pain have rarely attempted to restrict their estimates to the more severe episodes.

The aims of this study were to compare the influence on estimates of shoulder pain in a population survey of (a) different approaches to defining 'shoulder' and (b) restricting inclusion only to those with associated disability.

\section{Methods}

A cross sectional population survey was carried out to investigate the objectives. The initial survey was based on a self administered questionnaire. To determine the occurrence of disabling shoulder pain a subgroup of the responders were subsequently interviewed at home. The study population comprised a randomly selected sample of 500 patients aged 18-75 years from the age/sex register of a general practice in Stockport. A questionnaire enquiring about musculoskeletal symptoms was mailed to the sample and up to two reminders were sent to non-responders. These mailings took place over four months after which time 312 questionnaires had been returned; a response rate of $66 \%$ after adjusting for 29 people who had moved from the mailing address.

The questionnaire used a number of approaches to ascertain the presence of upper trunk symptoms that had lasted for more than 24 hours in the month before the survey. Four definitions of shoulder pain were derived from the answers to the questionnaire. The first definition was based on a direct question (fig 1). The second and third were based on line drawings on which the respondents were asked to shade any pain or aches experienced in the month before the survey. Markings in the areas shown in figure 1 were used for two definitions: (a) pain located in a restricted area in or around the shoulder complex and (b) pain felt in a wide region including the anterior chest, sterno-clavicular region, and between the scapulae (upper trunk). The fourth case definition was based on a direct question asking about symptoms in a pre-shaded area including the upper trunk and neck on a separate manikin (fig 2 ).

The prevalence of shoulder pain, according to each of the four definitions, was estimated among the 312 responders who had completed baseline questionnaires. The $95 \%$ confidence limits were calculated for the prevalence estimates. Positive responses to each of the four definitions of shoulder pain were compared with each other to examine their interrelations.

In the second stage a follow up interview of respondents to the cross sectional study was carried out. Those respondents indicating that they were suffering from 'current' shoulder symptoms, defined as pain on the day of interview, were asked to complete a short, 23 item, questionnaire enquiring about disability in daily living associated with such symptoms. This questionnaire was developed from the Functional Limitations Profile ${ }^{12}$ and was a slightly expanded version of a 22 item schedule validated in general practice and in the community. ${ }^{13}$ Topics covered by the questionnaire include domestic, recreational, and 
Table 1 Age and sex charactersitics of responders and non-responders to the survey

\begin{tabular}{|c|c|c|c|c|}
\hline & \multicolumn{2}{|c|}{ Responders $(n=312)$} & \multicolumn{2}{|c|}{ Non-responders $(n=188)$} \\
\hline & Number & $\%$ & Number & $\%$ \\
\hline \multicolumn{5}{|l|}{ Age group } \\
\hline $18-39$ years & 133 & 43 & 119 & 63 \\
\hline $40-59$ years & 109 & 35 & 52 & 28 \\
\hline $60+$ years & 70 & 22 & 17 & 9 \\
\hline \multicolumn{5}{|l|}{ Sex } \\
\hline Male & 139 & 45 & 106 & 56 \\
\hline Female & 173 & 55 & 82 & 44 \\
\hline
\end{tabular}

psychosocial problems relating to shoulder symptoms. In this version two additional items were added: 'because of my shoulder pain I cannot reach for things' and 'because of my shoulder pain I often moan and groan'. Two items referring to difficulty lying on the left and right side were replaced by a single item enquiring about difficulty lying on the painful side.

All respondents to the cross sectional survey were contacted and permission to interview them was sought. Of the 312 respondents to the survey, $232(74 \%)$ were successfully interviewed. The proportion of people reporting disabling shoulder symptoms was estimated based on these responders.

\section{Results}

Of the 312 people who completed baseline questionnaires 139 (45\%) were male and 173 $(55 \%)$ were female. The mean age of respondents was 44.7 years (range 18-73). There was a higher proportion of men and people in the younger age groups in non-responders to the survey (table 1 ).

In total $160(51 \%)$ people reported shoulder pain according to at least one definition. This one month period prevalence ranged from $31 \%$ to $48 \%$ across the four definitions (table 2 ). The lowest prevalence estimate was observed for the question asking respondents directly whether they had experienced symptoms in their shoulder(s). The highest prevalence was observed for the broadest definition asking about symptoms in a pre-defined area on a manikin (upper trunk and neck).

The degree of overlap of the four definitions was then analysed (fig 3). In total 84 people (27\% of all respondents) answered positively to all four of the case definitions. Only seven people who answered positively when asked directly if they had experienced symptoms in the shoulder (definition 1) did not indicate symptoms on the pain drawing in the 'restricted' area (definition 2) (fig 1). By contrast $65(30 \%)$ of those who answered negatively to the direct question either indicated symptoms on the pain drawing in the upper trunk region (definition 3) or answered positively to the question about symptoms in the upper trunk or neck region (definition 4) (fig 2). Only 19 (9\%) of those answering negatively to the direct question, however, indicated symptoms in the 'restricted' area on the pain drawing (definition 2), compared with 38 $(18 \%)$ indicating symptoms in the upper trunk region (definition 3) and 59 (27\%) symptoms in the upper trunk and neck region (definition 4).

The proportion of people interviewed who indicated having experienced symptoms in the past month (period prevalence) was higher than that seen in the original survey (table 2); $104(45 \%)$ interviewees marked symptoms in the shoulder complex on the blank manikin compared with 107 (34\%) of all the respondents to the original survey.

Limiting the definition to only include current symptoms (pain on the day of interview) with some associated disability (at least one item on the disability questionnaire being answered positively) restricted the point prevalence estimate to $20 \%(n=46)$.

\section{Discussion}

We have shown using a cross sectional survey design how different case definitions of shoulder pain lead to varying prevalence estimates of the disorder. It is not surprising that the style of question asked directly affects the proportion of people responding positively. Common sense would lead one to expect that as the anatomical area upon which the case definition is based is extended, so the prevalence estimate based on that definition will increase. Our observations have confirmed this.

Studies have found that imprecise perceptions of anatomical regions by respondents often lead to inaccurate reporting when respondents are asked directly about symptoms. ${ }^{14}$ We attempted to counter this by using definitions based on pain drawings that did not require respondents to make such a direct judgement. It was evident that the marking of symptoms in the 'restricted' area on the pain drawing did give different answers to a

Table 2 Prevalence of shoulder pain by definition

\begin{tabular}{|c|c|c|c|c|}
\hline \multirow[b]{2}{*}{ Definitions of shoulder pain } & \multicolumn{2}{|c|}{$\begin{array}{l}\text { Responders to cross } \\
\text { sectional survey }(n=312)\end{array}$} & \multicolumn{2}{|c|}{$\begin{array}{l}\text { Responders to cross sectional } \\
\text { survey and interview }(n=232)\end{array}$} \\
\hline & Number & $\%(95 \% C I)$ & Number & $\%(95 \% C I)$ \\
\hline \multicolumn{5}{|l|}{ Cross sectional survey } \\
\hline (A) Direct question - 'pain in shoulder(s)' & 96 & $31(26,36)$ & 74 & $32(26,38)$ \\
\hline (B) Pain marked on manikin - (shoulder complex) & 107 & $34(29,40)$ & 82 & $35(29,41)$ \\
\hline (C) Pain marked on manikin - (upper trunk) & 132 & $42(37,48)$ & 100 & $43(37,49)$ \\
\hline (D) Direct question - (pain in pre-defined area) & 150 & $48(43,54)$ & 116 & $51(45,57)$ \\
\hline \multicolumn{5}{|l|}{ Interview } \\
\hline (I) Pain marked on manikin - (shoulder complex) & - & - & 104 & $45(38,51)$ \\
\hline (II) Current pain & - & - & 73 & $32(26,37)$ \\
\hline (III) Current pain and associated disability ${ }^{\star}$ & - & - & 46 & $20(15,25)$ \\
\hline
\end{tabular}

ॠ At least one item of disability answered positively on the questionnaire. 'Pain' in (A) - (D) and in (I) refers to 'pain in the past month'- that is, period prevalence. 'Current pain' at interview in (II) and (III) refers to 'pain on the day of interview'-that is, point prevalence. 
(1) Direct question- pain in shoulder(s)

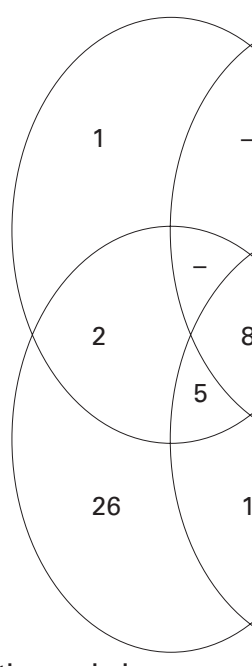

(4) Direct question- pain in pre-shaded area (upper trunk and neck)
(2) Pain shaded on manikin(shoulder complex) (shoulder complex)

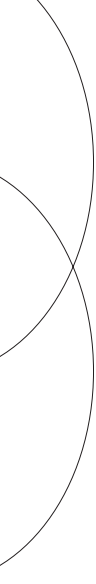

(3) Pain shaded on manikin(upper trunk region)

Figure 3 Overlap of definitions of shoulder pain from cross sectional survey.

direct question about symptoms. However most of those people answering positively to the direct question about shoulder symptoms $(92 \%)$ shaded an area exclusively involving the shoulder complex region (fig 1) on the pain drawing.

As the definition was broadened the prevalence estimate increased. In part this probably resulted from an increase in sensitivity due to the detection of symptoms originating in the shoulder complex but felt in adjacent areas. However the detection of symptoms unrelated to the shoulder is likely to have explained most of the increase. The associated drop in specificity would make the use of such a broad definition unsuitable for estimating the occurrence of shoulder symptoms.

There is no clinical 'gold standard' for defining shoulder pain. Studies often rely on specific diagnosis when defining shoulder pain..$^{15}$ Even in a clinical situation, however, such a diagnosis is very difficult ${ }^{16}$ and subject to considerable variation between clinicians. ${ }^{17}$ The good repeatability found in our study between self reported 'shoulder pain' (definition 1) and pain shaded on a manikin (definition 2) suggests that either would be a reasonable basis for an epidemiological definition in the absence of a 'gold standard'. The extensive work validating the use of pain drawings in low back pain ${ }^{1418}$ and the advantage that such drawings can be classified independently and can be used to identify pain outside the shoulder area would lead us to recommend this method (definition 2), rather than self reported shoulder pain, for epidemiological studies.

For all the definitions used in the cross sectional survey we found a high prevalence. This is consistent with other studies of the prevalence of musculoskeletal symptoms ${ }^{19}$ and is a general feature of symptom based surveys. The follow up study allowed us to categorise this group more distinctively according to current shoulder pain and current shoulder pain with associated disability. When the inclusion criteria were restricted to current episodes of shoulder pain (that is, pain on the day of interview-point prevalence) a lower prevalence estimate was observed with the probable exclusion of the more transient symptoms. Even this point prevalence estimate, however, represented one third of the responders to the interview. A further restriction of the case definition to current shoulder symptoms in association with some disability resulted in a reduction of the point prevalence estimate to $20 \%$. This probably reflects the exclusion of the more minor episodes of shoulder pain. It would be reasonable to assume that, given that items of disability were specifically related to shoulder symptoms, pain referred from other regions that are unlikely to have affected shoulder function would not be included in this case definition. It is unlikely that the reduction in prevalence seen when including disability items resulted from an overall improvement between the cross sectional survey and follow up because a higher prevalence of shoulder symptoms in the past month was observed at interview than in the original survey using an identical definition.

Using responses to the disability questionnaire to increase the specificity of the case definition depends on the respondents' self assessment of associated limitations of activities of daily living. An alternative approach is to restrict the case definition to shoulder pain with associated restriction of movement as assessed at clinical examination. In a separate study, however, we found that restriction in several planes of upper limb movement was not useful in defining shoulder pain episodes. ${ }^{20}$

There are a number of methodological issues to be considered. It is possible that the way in which the questions were asked would have directly influenced the prevalence estimates obtained. Firstly, answers to the questions might have been influenced by the fact that they were included as part of the same questionnaire. If such a phenomenon was taking place one would expect the different questions to be mutually consistent. However we observed an increasing proportion of positive answers as the definition of shoulder pain was broadened.

Secondly, questions were always asked in the same order and this might have contributed to the observed prevalence estimates, with positive answers to initial questions influencing positive answers to subsequent questions. An alternative approach would have been to ask the questions in a random order. In this study, however, questions were deliberately asked in an order going from the general (a statement about shoulder pain included in a section about general aches and pains) to the specific (shoulder symptoms indicated by a pre-shaded manikin) to reduce response bias.

The sampling frame used in this study was an age/sex register from a single general practice in south Manchester. This would limit the generalisability of our prevalence estimates to wider populations. Although the estimates of 
prevalence might vary in different populations, it is probable that their interrelations would be similar.

From the original random sample of 500 people only 312 responded to the cross sectional survey questionnaire. We compared general practice consultation data of nonresponders with that of a matched sample of responders $^{21}$ and found that people who responded were more likely to have consulted their doctor for shoulder pain in the two years before the survey. This is in line with many studies of symptoms that have found that those with the problem under investigation are more likely to respond. ${ }^{22}$ It suggests that prevalence estimates of shoulder symptoms based on the responders to the cross sectional survey may be an overestimate of the prevalence in the general population. The main purpose of this study, however, was to compare responses to different case definitions within the study group and such bias will only become important when extrapolating prevalence estimates to the general population.

The sample of people followed up to interview were a more select group with only $74 \%$ of the responders to the cross sectional survey being successfully interviewed. The increased period prevalence (pain in the past month) seen when identical definitions to those used in the original survey were adopted at interview suggest interview responders were more likely to be those with symptoms. We found similar prevalence estimates, however, in the original survey in those people who were subsequently interviewed compared with all baseline responders (table 2). This suggests that non-responders at interview were likely to be those whose symptoms had resolved since the original survey. However, again this does not affect the within group comparisons used to establish whether disability can be used to improve case definition.

In summary, using a pain drawing based definition with case ascertainment restricted to an area in and around the shoulder complex is recommended for surveys assessing the occurrence of shoulder symptoms in the general population. To solve the problem of the poor specificity associated with symptom based definitions it is useful to incorporate an additional classification to restrict the definition to more disabling problems.

This study was supported by the Arthritis and Rheumatism Council. We would like to thank Ms Michelle Martin, Dr
Christopher Martin, Dr Richard Hardman, Dr Frances Nield, and Dr Christopher Marshall for their assistance with the soft issue rheumatism studies. In addition, we would like to thank staff and patients at the Heaton Norris Health Centre.

1 Office of Population Census and Surveys (OPCS), The Royal College of General Practitioners, Department of
Health and Social Security. Morbidity Statistics from general Health and Social Security. Morbidity Statistics from general
practice, 3rd national survey 1981-82. London:HMSO, practice,

2 Hasvold T, Johnsen R. Headache and neck or shoulder pain - frequent and disabling complaints in the general population. Scand J Prim Health Care 1993;11:219-24.

3 Nygren A, Berglund A, Von Koch M. Neck and shoulder pain, an increasing problem. Strategies for using insurance 32:107-12.

4 Bjelle A. Epidemiology of shoulder problems. Baillieres Clin Rheumatol 1989;3:437-51

5 Cyriax J. Textbook of orthopaedic medicine. Diagnosis of soft tissue lesions. Vol 1. 6th ed. London: Bailliere Tindall, 1976.

6 Buchbinder R, Goel V, Bombardier C, Hogg-Johnson S. Classification systems of soft tissue disorders of the neck and upper limb: do they satisfy methodological guidelines? J Clin Epidemiol 1996;49:141-9.

7 Cunningham LS, Kelsey JL. Epidemiology of musculoskeletal impairments and associated disability. Am J Public Health 1984;74:574-9.

8 Takala J, Sievers K, Klaukka T. Rheumatic symptoms in the middle-aged population in southwestern Finland. Scand J middle-aged population in sou
Rheumatol 1982;47:15-29.

9 Spangfort E. Clinical aspects of neck-and-shoulder pain. Scand J Rehab Med Suppl 1995;32:43-6.

10 Andersson HI, Ejlertsson G, Leden I, Rosenberg C. Chronic pain in a geographically defined general population: studies of differences in age, gender, social class and pain localisation. Clin J Pain 1993;9:174-82.

11 Chard MD, Hazleman R, Hazleman BL, King RH, Reiss BB. Shoulder disorders in the elderly: a community survey. Arthritis Rheum 1991;34:766-9.

12 Patrick DL, Peach H. Disablement in the community. Oxford: Oxford University Press, 1989

13 Croft P, Pope D, Zonca M, O'Neill T, Silman A. Measurement of shoulder related disability: results of a validation study. Ann Rheum Dis 1994;53:525-8.

14 Raspe HH. Back pain. In: Silman AJ, Hochberg MC, eds. Epidemiology of the rheumatic diseases. Oxford: Oxford University Press, 1993:330-74.

15 Jacobsson L, Lindgarde F, Manthorpe R. The commonest rheumatic complaints of over six weeks' duration in a twelve-month period in a defined Swedish population. Prevalence and relationships. Scand J Rheumatol 1989; 18:353-60.

16 Cailliet R. Shoulder pain. 3rd ed. Philadelphia: F A Davis, 1991.

17 Buchanan WW, Kahn MF, Laine V, Rodnan GP, Scott JT, Zvaifer NJ. Clinics in rheumatic diseases. Measurement of joint movement. London: W B Saunders, 1982.

18 Ohlund C, Eek C, Palmbald S, Areskoug B, Nachenson A, et al. Quantified pain drawing in subacute low back pain: validation in a non-selected outpatient industrial sample. Spine 1996;21;1021-31.

19 Papageorgiou AC, Croft PR, Ferry S, Jayson MIV, Silman AJ. Estimating the prevalence of low back pain in the general population: evidence from the South Manchester back pain survey. Spine 1995;20:1889-94.

20 Pope DP, Croft PR, Pritchard CM, Macfarlane GJ, Silamn AJ. The frequency of restricted range of movement in individuals with self-reported shoulder pain: results from a population based survey. Br J Rheumatol 1996;35:113741 .

21 Pope D, Croft P. Surveys using general practice registers: who are the non-responders? J Public Health Med who are the

22 Locker D, Slade GD, Leake JL. The response rate problem in oral health surveys of older adults in Ontario. Can J Public Health 1990;81:210-4. 